\title{
Информатика и
}

\section{информационные технологии}

УДК 616.12

\section{ОЦЕНКА ХАОТИЧНОСТИ ФОРМЫ ФРАГМЕНТОВ ОДНОКАНАЛЬНОЙ ЭЛЕКТРОКАРДИОГРАММЫ}

\author{
Л.С. Файнзильберг ${ }^{1}$, К.Б. Ориховская ${ }^{1}$, И.В. Ваховский ${ }^{2}$ \\ ${ }^{1}$ Международный научно-учебный центр информационных технологий и \\ систем НАН Украины и МОН Украины (2. Киев) \\ ${ }^{2}$ Национальный технический университет Украины «КПИ» (2. Киев)
}

Проведен сравнительный анализ различных подходов к оценке степени хаотичности формы элементов ЭКГ. Предложен ряд усовершенствований известных методов. Представлена структура инструментальной системы, ориентированной на выбор и настройку вычислительных алгоритмов, обеспечивавших оценку хаотичности формы элементов ЭКГ. Представлены результаты экспериментов, проведенных с помощью системы на модельных и реальных данных.

Ключевье слова: информационная технология, хаотичность биологического сигнала, форма фрагментов ЭКГ, сердечный ритм.

Проведено порівняльний аналіз різних підходів до оцінки ступеня хаотичності форми елементів ЕКГ. Запропоновано ряд удосконалень відомих методів. Представлено структуру інструментальної системи, орієнтованої на вибір і налаштування обчислювальних алгоритмів, що забезпечують оцінку хаотичності форми елементів ЕКГ. Надано результати експериментів, проведених за допомогою системи на модельних і реальних даних.

Ключові слова: інформаційна технологія, хаотичність біологічного сигналу, форма фрагментів ЕКГ, серцевий ритм.

\section{ВВЕДЕНИЕ}

При изучении динамики поведения сложных медико-биологических систем все большее внимание привлекают методы теории хаоса и синергетики [1], позволяющие более полно раскрыть и проанализировать механизмы функционирования живой сложноорганизованной системы. Эти методы нашли применение и в кардиологии для оценки хаотичности сердечного ритма, который несет информацию о функциональном состоянии всех звеньев регулирования жизнедеятельности человека как в норме, так и при различных патологиях [2-4]. Такая оценка обычно проводится по динамическому ряду продолжительностей сердечных циклов ( $R-R$ интервалов), которые определяются в процессе регистрации 
электрокардиограммы (ЭКГ) [5].

В то же время нельзя полноценно судить о функциональном состоянии самого сердца как основного системообразующего органа лишь на основе анализа хаотичности ритма сердца. Поэтому представляет интерес развитие методов и инструментальных средств синергетики в кардиологии, ориентированных на оценку хаотичности не только $R-R$ интервалов, но и других диагностически ценных показателей ЭКГ, несущих информацию о форме фрагментов ЭКГ от цикла к циклу - зубцов $P, Q, R, S, T$, интервалов $P-Q, \quad Q-T$ и сегмента $S T$. Следует заметить, что результаты исследований хаотичности таких показателей практически отсутствуют в доступной научной литературе.

Цель - создать инструментальную систему для исследования хаотичности формы элементов одноканальной ЭКГ на основе различных энтропийных оценок и провести их сравнительный анализ на модельных и реальных данных.

\section{АНАЛИЗ ИЗВЕСТНЫХ ПОДХОДОВ К ОЦЕНКЕ ХАОТИЧНОСТИ ВРЕМЕННЫХ РЯДОВ}

В основе многих математических методов исследования хаотичности динамических рядов лежит известная формула Шенноновской энтропии [6]

$$
H=-\sum_{l=1}^{n} p_{l} \log _{2} p_{l},
$$

предложенная для оценки неопределенности системы, находящейся в одном из $n$ состояний с вероятностями $p_{l}, l=1, \ldots, n$. Чем больше величина $H$, тем дальше система находится от упорядоченного состояния, причем максимальное значение энтропии Шеннона достигается, когда все $p_{l}$ равны, т.е. состояния системы равновозможны [7].

Для анализа хаотичности конечного временного ряда

$$
A=a_{1}, a_{2}, \ldots, a_{N}
$$

элементы которого представляют собой скалярные величины $a_{i} \in\left[a_{i}^{-}, a_{i}^{+}\right]$, $i=1, \ldots, N$, принадлежащие заданным интервалам, в ряде работ, в частности, в [8], предлагается прямо воспользоваться формулой (1), оценив вероятности (частоты) попадания значений ряда (2) в указанные интервалы.

Однако величина (1) инвариантна относительно перестановок элементов ряда (2). Поэтому при непосредственном использовании формулы (1) оценивается не хаотичность последовательности (2), а неопределенность случайной величины, порождающей эту последовательность, что не одно и то же.

Например, две последовательности - регулярная

$$
A_{1}=1,0,1,0,1,0,1,0,1,0,1,0,1,0
$$


и хаотичная

$$
A_{2}=0,1,0,0,0,0,1,1,0,1,1,1,0,1,
$$

будут иметь одинаковые значения энтропии $H=1$.

Оценить хаотичность временного ряда при непосредственном использовании формулы (1) можно лишь в том случае, если последовательность (2) обрабатывать по частям (в окнах) или оценивать изменение энтропии по ходу накопления данных

$$
H(k)=-\sum_{i=1}^{n} p_{i}(k) \log _{2} p_{i}(k), k \leq N .
$$

Существуют и другие подходы к решению данной задачи, например использование условной энтропии [9]. Для этого последовательность (2) разбивается на подпоследовательности (паттерны)

$$
x(i)=[a(i), a(i+1), \ldots, a(i+m-1)], i=1, \ldots, N-m+1
$$

с размерностью вложения $m$, оцениваются вероятности (частоты) появления конкретных паттернов и вычисляется условная энтропия $E(m \mid m-1)$ как приращение Шенноновской энтропии при переходе от паттернов с размерностью $m-1 \kappa m$, т.е.

$$
E(m \mid m-1)=E(m)-E(m-1)=-\sum_{i=1}^{N-m+1} p_{m} \ln p_{m}+\sum_{i=1}^{N-m+2} p_{m-1} \ln p_{m-1} .
$$

Согласно [10], переход от последовательности скалярных величин (2) к векторам (4) можно интерпретировать как переход в фазовое (псевдофазовое) пространство, в котором и проводится дальнейший анализ.

Для более полного анализа сложности медико-биологических систем получили известность другие энтропийные оценки, в частности, аппроксимационная энтропия ApEn (Approximation Entropy) [11], которую в русскоязычной литературе называют энтропия подобия [12].

При ее вычислении исходная последовательность также разбивается на паттерны (4), близость которых в фазовом пространстве оценивается расстоянием

$$
d[x(i), x(j)]=\max _{k=1, \ldots, m}\{|a(i+k-1)-a(j+k-1)|\}
$$

между всеми парами $x(i)$ и $x(j), i=1, \ldots, N-m+1, j=i, \ldots, N-m+1$. Далее оцениваются вероятности (частоты) появления в последовательности (2) таких пар паттернов, расстояние между которыми не превышает заданный порог $d_{0}[13]$. Для этого определяются величины

$$
C_{r}^{(m)}(i)=\frac{U^{(m)}(i)}{N-m+1}
$$

в которых $U^{m}(i)$ - количество значений $d[x(i), x(j)]$, удовлетворяющих условию

$$
d[x(i), x(j)] \leq d_{0}, \quad j=1, \ldots, N-m+1,
$$


или в эквивалентной форме записи

$$
C_{r}^{(m)}(i)=\frac{1}{N-m+1} \sum_{j=1}^{N-m+1} \Theta\left(d_{0}-d[x(i), x(j)]\right),
$$

где

$$
\Theta(\eta)=\left\{\begin{array}{l}
1, \text { если } \eta \geq 0 \\
0, \text { если } \eta<0
\end{array}\right.
$$

- функция Хевисайда.

Используя выражения (7) или (9), можно вычислить величину

$$
\theta^{(m)}(r)=\frac{1}{N-m+1} \sum_{i=1}^{N-m+1} \ln C_{r}^{(m)}(i),
$$

которую также принято называть безусловной энтропией [9].

Аппроксимационная энтропия определяется как приращение безусловной энтропии (11) при переходе от последовательности паттернов длиной $m$ к последовательности длиной $m+1$ по формуле

$$
A p E n=\theta^{(m)}(r)-\theta^{(m+1)}(r) .
$$

Обычно [14] для расчета используют приближенную формулу

$$
A p E n=\frac{1}{N-m} \sum_{i=1}^{N-m} \ln \frac{C_{r}^{m}}{C_{r}^{m+1}} .
$$

Значения аппроксимационной энтропии для регулярной и хаотической последовательностей $A_{1}$ и $A_{2}$ будут соответственно равны $\operatorname{ApEn}\left(A_{1}\right)=0,08$ и $\operatorname{ApEn}\left(A_{2}\right)=0,4$.

Следует заметить, что оценка (13) является смещенной оценкой аппроксимационной энтропии, значения которой асимптотически возрастают с увеличением количества $N$ элементов временного ряда [12]. Для устранения этого недостатка в работе [15] предложена небольшая модификация метода вычисления $A p E n$, которая предусматривает использование величин

$$
C_{r}^{(m)}(i)=\frac{\sigma+U^{(m)}(i)}{N-m+1},
$$

числитель которых, в отличие от (7), содержит фиксированную добавку $\sigma>0$, а в условие (8) вводится дополнительное ограничение $i \neq j$.

Дальнейшая модификация аппроксимационной энтропии позволила предложить энтропию шаблонов SampEn (Sample entropy) [16], при вычислении которой также используется ограничение $i \neq j$, а саму энтропию определяет выражение

$$
\text { SampEn }=-\ln \frac{U^{m+1}(r)}{U^{m}(r)}=\ln U^{m}(r)-\ln U^{m+1}(r) .
$$


Такая оценка, в отличие от (12), является несмещенной, а ее значение практически не зависит от количества элементов временного ряда $N$ [16].

Еще одним средством оценки хаотичности временных рядов является перестановочная энтропия PE (Permutation Entropy) [17], основанная на анализе паттернов (4) не с точки зрения расстояния между ними в фазовом пространстве, а с точки зрения их формы (класса).

Для классификации паттернов каждому его элементу $a(i)$ присваивается метка, характеризующая уровень элемента по отношению к соседнему, например, метки $0,1,2$. Тогда при $m=3$ можно различать 3 ! (шесть) классов паттернов $\pi_{1}, \pi_{2}, \ldots, \pi_{6}$, образованных тройкой последовательных значений соответствующего уровня (рис.1).

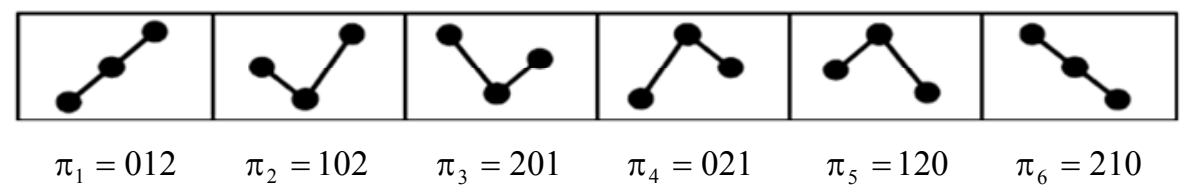

Рис 1. Классы паттернов перестановочной энтропии при $m=3$.

Перестановочная энтропия вычисляется по формуле:

$$
P E(m)=-\sum_{i=1}^{N-m+1} p\left(\pi_{i}\right) \log _{2} p\left(\pi_{i}\right), i=1, \ldots m !,
$$

в которой $p\left(\pi_{i}\right)$ - частота появления паттерна $i$-го класса при обработке исходной последовательности (2) скользящим окном из $m$ точек.

Значения перестановочной энтропии (16) для регулярной и хаотической последовательностей $A_{1}$ и $A_{2}$ соответственно равны $P E\left(A_{1}\right)=1 \quad$ и $\operatorname{PE}\left(A_{2}\right)=1,96$.

В том случае, когда элементы временного ряда (2) образуют не скалярные величины $a_{i}$, а $R$-мерные вектора $\vec{a}_{i}=\left(a_{i 1}, \ldots, a_{i R}\right), \quad$ для интегральной оценки хаотичности такого ряда используют корреляционный интеграл [18]. Для его вычисления компоненты векторов $\vec{a}_{i}=\left(a_{i 1}, \ldots, a_{i R}\right)$ предварительно нормируются и дальнейшая обработка осуществляется в фазовом пространстве с нормированными координатами $a_{i 1}^{*}, \ldots, a_{i R}^{*}$.

Оценку корреляционного интеграла $C\left(d_{0}, N\right)$ при конечном числе $N$ наблюдений дает выражение:

$$
C\left(d_{0}, N\right)=\frac{1}{N^{2}} \sum_{m, l=1}^{N} \Theta\left(d_{0}-d\left(\vec{a}_{l}, \vec{a}_{m}\right)\right),
$$

в котором $d\left(\vec{a}_{l}, \vec{a}_{m}\right)$ - евклидово расстояние между $l$-м и $m$-м векторами в $R$-мерном фазовом пространстве, $d_{0}$ - заданный порог, а $\Theta(\cdot)-$ функция Хевисайда, определяемая выражением (10). 
Тем самым оценивается средняя вероятность того, что нормированные вектора $\quad \vec{a}_{i}=\left(a_{i 1}, \ldots, a_{i R}\right), \quad i=1, \ldots, N, \quad$ образующие $\quad$ обрабатываемую последовательность, окажутся на расстоянии, не превышающем заданный порог $d_{0}$.

Известно [19, 20], что для фрактальных (самоподобных) множеств характерна зависимость:

$$
\lim _{d_{0} \rightarrow 0} C\left(d_{0}, N\right)=d_{0}^{D}
$$

где $D$ - размерность аттрактора (Fractal dimension), которая также оценивает хаотичность временных сигналов в $R$-мерном фазовом пространстве [21].

Из (18) следует, что по вычисленному корреляционному интегралу (17) можно определить размерность аттрактора по формуле:

$$
D=\lim _{d_{0} \rightarrow 0} \frac{\log \left(C\left(d_{0}\right)\right)}{\log d_{0}} .
$$

На основании (19) заключаем, что фрактальную размерность $D$ можно определить по наклону прямой линии регрессии, построенной по экспериментальным данным в координатах $\ln C\left(d_{0}\right), \ln d_{0}$.

Для удобства выразим эту зависимость в явном виде:

$$
D=\frac{N \sum_{i=1}^{N} \log d_{0}(i) \cdot \log \left(C\left(d_{0}(i)\right)\right)-\sum_{i=1}^{N} \log d_{0}(i) \sum_{i=1}^{N} \log \left(C\left(d_{0}(i)\right)\right)}{N \sum_{i=1}^{N}\left(\log d_{0}(i)\right)^{2}-\left(\sum_{i=1}^{N} \log d_{0}(i)\right)^{2}} .
$$

Известен и другой подход к вычислению фрактальной размерности [21], основанный на размерности Минковского $D_{b c}$ (Box-counting dimension). Метод заключается в определении минимального числа $M(d)$ «ячеек» фазового пространства диаметром $d \rightarrow 0$, которыми можно «накрыть» исходное множество точек.

После ряда преобразований получим в явном виде окончательную формулу для вычисления размерности Минковского как угловой коэффициент прямой линии регрессии, построенной по экспериментальным точкам в координатах $\ln M(d), \ln d_{0}(i)$ :

$$
D_{b c}=\frac{N \sum_{i=1}^{N} \ln d_{0}(i) \cdot \ln M(d(i))-\sum_{i=1}^{N} \ln d_{0}(i) \sum_{i=1}^{N} \ln M(d(i))}{N \sum_{i=1}^{N}\left(\ln d_{0}(i)\right)^{2}-\left(\sum_{i=1}^{N} \ln d_{0}(i)\right)^{2}} .
$$

Еще одним достаточно простым и эффективным методом анализа нестационарных временных рядов является так называемый $R / S$ - анализ, основанный на вычислении индекса Херста $H_{0}$ (Hurst exponent) [22] по модифицированному временному ряду 


$$
z_{i}=\sum_{i=1}^{k}\left(a_{i}-a_{c p}\right), i=1, \ldots, k,
$$

где $k \leq N, a_{c p}-$ среднее значение элементов в исходной последовательности (2) из $N$ элементов, или же по другому модифицированному ряду в виде логарифма отношений:

$$
z_{i}=\log \left(\frac{a_{i+1}}{a_{i}}\right), \quad i=1,2, \ldots, N-1 .
$$

Для вычисления индекса Херста достаточно определить размах

$$
R^{(k)}=\max _{i=1, \ldots, k}\left(z_{i}\right)-\min _{i=1, \ldots, k}\left(z_{i}\right)
$$

и стандартное отклонение

$$
S^{(k)}=\sqrt{\frac{1}{k-1} \sum_{i=1}^{k}\left(a_{i}-a_{c p}\right)^{2}}
$$

при некоторых фиксированных значениях $k \leq N$.

Установлено [22], что для большинства стохастических рядов выполняется соотношение:

$$
R^{(k)} / S^{(k)}=L k^{H_{0}},
$$

т.е. нормированный размах пропорционален степени $k$, где $H_{0}$ - показатель Херста, а $L$ - некоторая константа.

Для определения показателя Херста в удобной форме прологарифмируем уравнение (25)

$$
\log \left(R^{(k)} / S^{(k)}\right)=\log L+H_{0} \log k
$$

и, также как в предыдущих случаях, выразим значение индекса Херста в виде углового коэффициента прямой линии регрессии (26), которая наилучшим образом аппроксимирует экспериментальные точки $\log \left(R^{(k)} / S^{(k)}\right)$ и $\log k$, найденные при различных фиксированных значениях $k \leq N$.

В результате, после ряда очевидных преобразований, основанных на обработке методом наименьших квадратов значений ряда при всех $k=1, \ldots, N$, получим

$$
H_{0}=\frac{\log k \sum_{k=1}^{N} \log k \log \left(R^{(k)} / S^{(k)}\right)-\sum_{k=1}^{N} \log k \sum_{k=1}^{N} \log \left(R^{(k)} / S^{(k)}\right)}{\log k \sum_{k=1}^{N}(\log k)^{2}-\left(\sum_{k=1}^{N} \log k\right)^{2}} .
$$

Разумеется, приведенный анализ существующих методов не претендует на полноту, тем более, что методы оценки хаотичности временных рядов постоянно развиваются. Мы рассмотрели лишь наиболее популярные подходы и получили окончательные формулы, удобные для практического применения. 
На основании проведенного анализа можно сделать вывод, что современная наука располагает эффективными методами и вычислительными алгоритмами, которые позволяют с различных сторон оценить степень хаотичности временных рядов, порождаемых медико-биологическими системами.

В то же время анализ доступной литературы не дает полной ясности в главном вопросе: какой из методов целесообразно применить при решении конкретной прикладной задачи. К тому же, каждый вычислительный алгоритм содержит параметры, от выбора значений которых зависит полученный результат. Однако не существует формальных подходов к оптимальной настройке вычислительных алгоритмов, например, к выбору оптимальных значений порога $d_{0}$ в процедурах (8), (9), (17) или же размерности вложения $m$ при переходе от последовательности (2) к паттернам (4). Отсюда следует, что при построении конкретных прикладных систем выбор подходящего вычислительного алгоритма и его настройку, решение о необходимости его модификации или разработки альтернативного алгоритма приходится принимать экспериментальным путем на модельных и реальных данных (рис. 2).

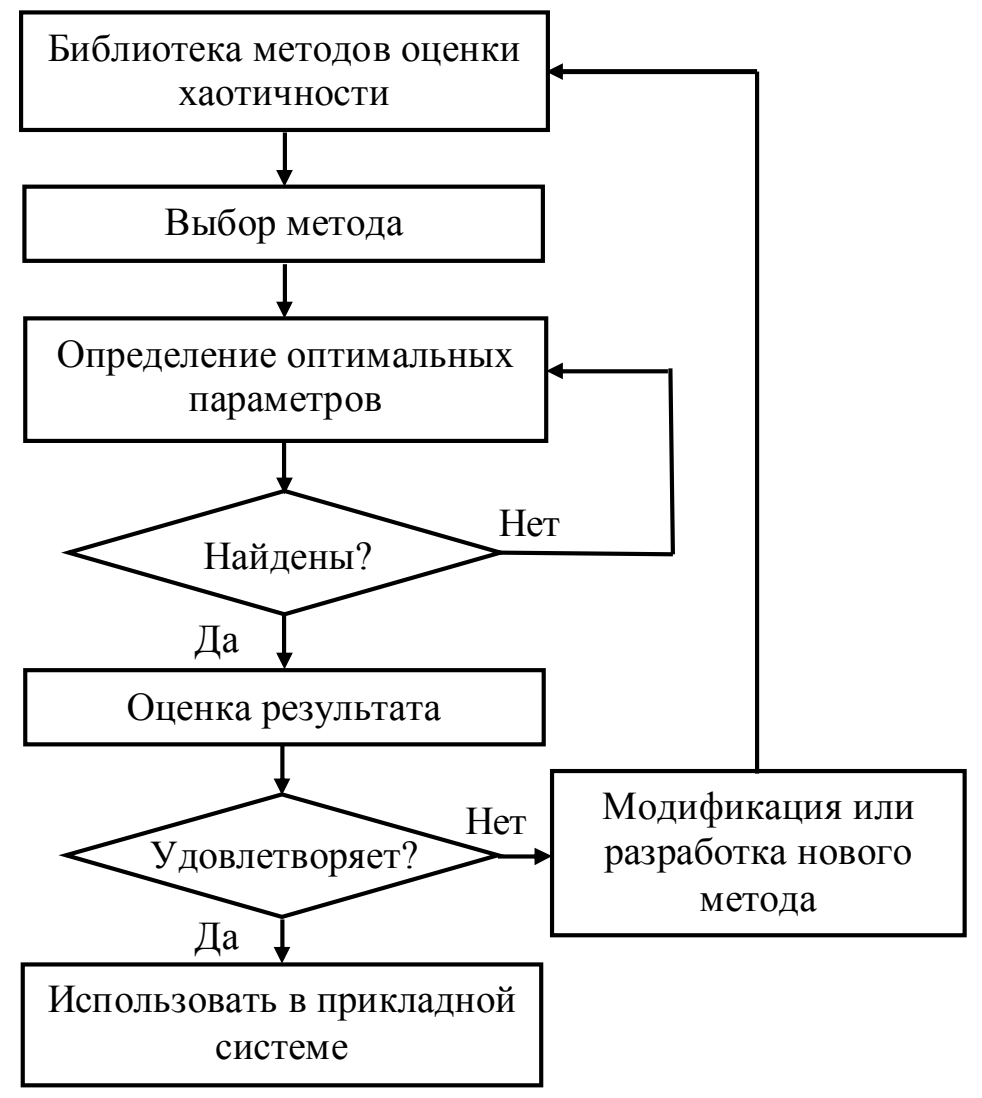

Рис. 2. Идея выполнения экспериментов 
Рассмотрим предлагаемый подход к построению инструментальной системы, обеспечивающей проведение таких экспериментов на примере оценки хаотичности формы фрагментов одноканальной ЭКГ.

\section{ОБЩАЯ ИДЕЯ И СТРУКТУРА ИНСТРУМЕНТАЛЬНОЙ СИСТЕМЫ}

Для реализации отдельных этапов предлагаемой ИТ обработки ЭКГ разработана инструментальная система (рис. 3), построенная на основе отечественного портативного электрокардиографа ФАЗАГРАФ $\Phi^{\circledR}$ с оригинальным сенсором с пальцевыми электродами [24].

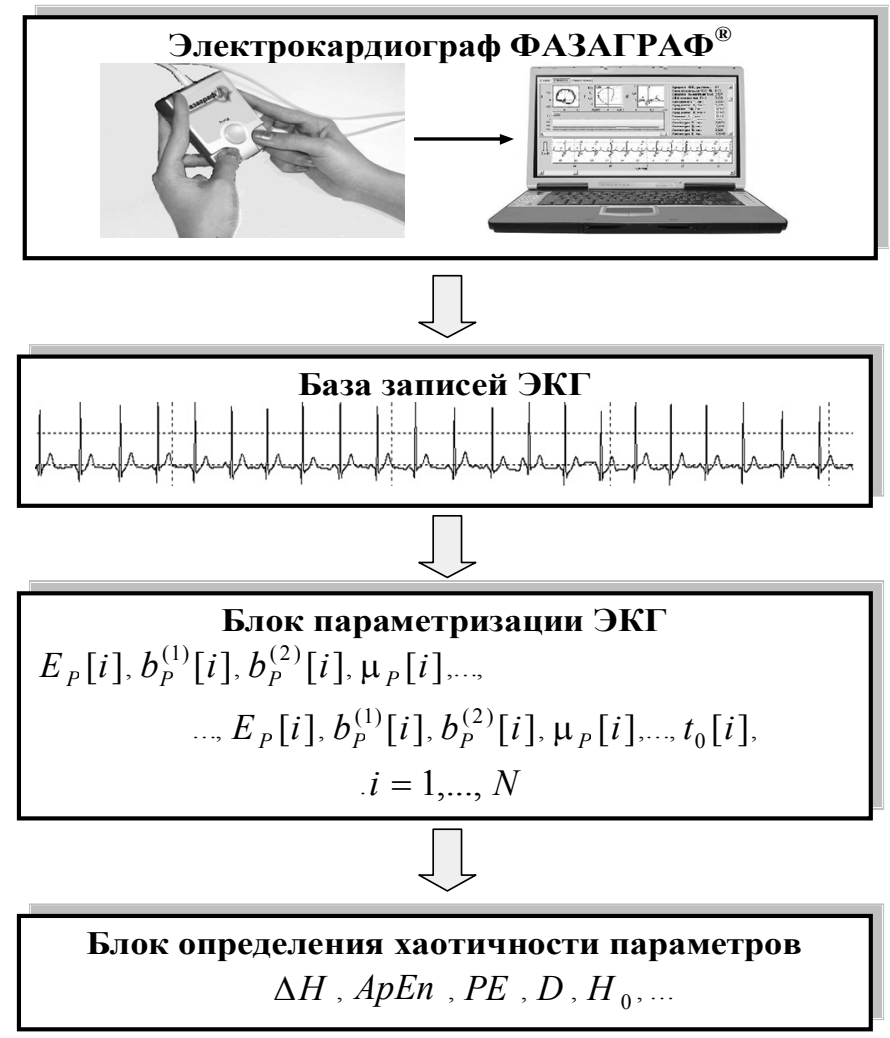

Рис. 3. Структура инструментальной системы

Сенсор позволяет надежно регистрировать ЭКГ первого стандартного отведения без снятия одежды не только в клинических, но и в полевых условиях, при занятиях спортом, непосредственно на рабочем месте и в домашних условиях. Оцифрованные записи ЭКГ через USB порт вводятся в компьютер и сохраняются в базе данных (БД) совместно с необходимыми персональными данными обследуемого.

Выбранная запись ЭКГ поступает на вход модуля параметризации, который обеспечивает переход от дискретных значений сигнала к массивам параметров, характеризующих форму зубцов $P, Q, R, S, T$ и сегмента $S T$ на последовательности сердечных циклов. 
С этой целью каждый из указанных фрагментов, представленный последовательностью значений $z_{k}\left(t_{m}\right), k \in\{P, Q, R, S, S T, T\}$ в дискретные моменты времени $t_{m}, \quad m=1, \ldots, M_{k}$, аппроксимируется несимметричной гауссовой функцией:

$$
\varphi_{k}(t)=E_{k} \exp \left[-\frac{\left(t-\mu_{k}\right)^{2}}{2\left[b_{k}(t)\right]^{2}}\right], k \in\{P, Q, R, S, S T, T\},
$$

в которой параметры $E_{k}$ и $\mu_{k}$ определяют значения амплитуд и моментов времени, когда $k$-й фрагмент принимает максимальное значение при $E_{k}>0$ или минимальное значение при $E_{k}<0$, а параметры

$$
b_{k}(t)= \begin{cases}b_{k}^{(1)} & \forall t \leq \mu_{k}, \\ b_{k}^{(2)} & \forall t>\mu_{k},\end{cases}
$$

при $b_{k}^{(1)} \neq b_{k}^{(2)}$ позволяют описать несимметричные фрагменты, например, несимметричный зубец $T$, если $b_{T}^{(1)} \neq b_{T}^{(2)}$ (рис 4).

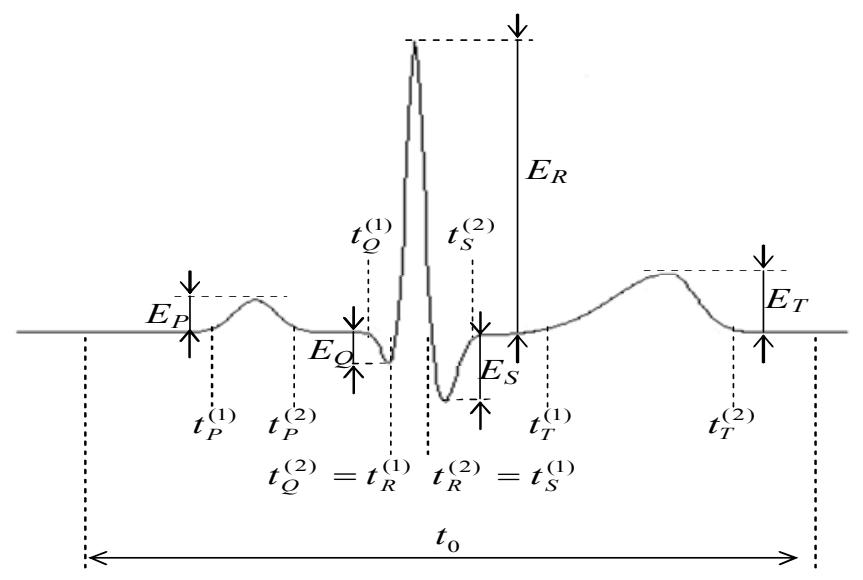

Рис. 4. Параметры формы фрагментов цикла ЭКГ

Оптимальные значения параметров $E_{k}, \mu_{k}, b_{k}^{(1)}, b_{k}^{(2)}$ для каждого $k$-го фрагмента $k \in\{P, Q, R, S, S T, T\}$ определяются по критерию минимума суммы квадратов уклонений функции (28) от дискретных значений $z_{k}\left(t_{m}\right)$, $m=1, \ldots, M_{k}$ этого фрагмента, т.е. удовлетворяют критерию:

$$
C r_{k}=\sum_{m=1}^{M_{k}}\left[E_{k} \exp \left[-\frac{\left(t_{m}-\mu_{k}\right)^{2}}{2\left[b_{k}\left(t_{m}\right)\right]^{2}}-z_{k}\left(t_{m}\right)\right]^{2} .\right.
$$

В результате каждый $i$-й цикл реальной ЭКГ продолжительностью $t_{0}[i]$ порождает 25 параметров $E_{P}[i], b_{P}^{(1)}[i], b_{P}^{(2)}[i], \mu_{P}[i], \ldots, E_{T}[i], b_{T}^{(1)}[i]$, 
$b_{T}^{(2)}[i], \quad \mu_{T}[i], t_{0}[i]$, которые представляют собой компоненты векторов $\vec{a}_{i}=\left(a_{i 1}, \ldots, a_{i R}\right), i=1, \ldots, N, R=25$ временного ряда (2).

Для анализа хаотичности формы фрагментов ЭКГ достаточно применить к последовательностям $E_{P}[i], b_{P}^{(1)}[i], \quad b_{P}^{(2)}[i], \mu_{P}[i], \ldots, E_{T}[i], b_{T}^{(1)}[i]$, $b_{T}^{(2)}[i], \mu_{T}[i], t_{0}[i], i=1, \ldots, N$ любой из рассмотренных выше методов, которые программно реализованы в соответствующем блоке инструментальной системы.

Оценка хаотичности диагностических признаков, сосредоточенных на информативных фрагментах, также представляет интерес. В инструментальной системе обеспечивается переход от массивов первичных параметров $E_{P}[i], b_{P}^{(1)}[i], b_{P}^{(2)}[i], \mu_{P}[i], \ldots, E_{T}[i], b_{T}^{(1)}[i], b_{T}^{(2)}[i], \mu_{T}[i]$, $t_{0}[i]$ к массивам диагностических признаков $E_{P}[i], E_{Q}[i], E_{S T}[i], E_{T}[i]$, $P Q[i], Q T[i], \Delta_{Q}[i], \Delta_{Q R S}[i], \beta_{T}[i], t_{0}[i]$, степень хаотичности которых от цикла к циклу также оценивается средствами инструментальной системы.

Выбор исходных данных, метода оценки хаотичности и настройка параметров вычислительных процедур осуществляется в интерактивном режиме с использованием элементов управления рабочего окна инструментальной системы (рис. 5). Результат обработки представляется в текстовом и графическом видах.
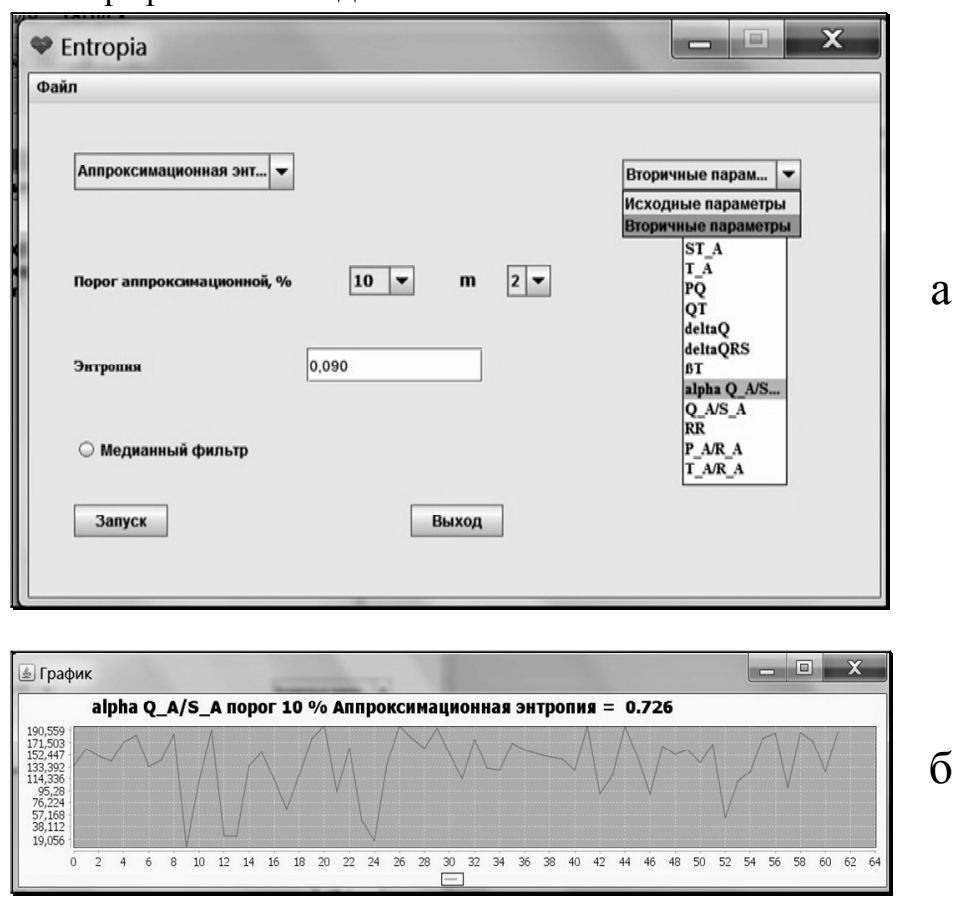

Рис. 5. Рабочие окна инструментальной системы: диалоговое окно (а); окно отображения графика обрабатываемого массива (б) 
При реализации процедур оценки степени хаотичности был внесен ряд усовершенствований в описанные выше традиционные методы. В частности, в инструментальной системе реализован оригинальный вычислительный алгоритм оценки перестановочной энтропии по последовательным значениям $a_{i-1}, \quad a_{i}, \quad a_{i+1}, \quad i=2, \ldots N-1$ временного сигнала (2), который на фоне допустимых искажений автоматически относит обрабатываемую тройку значений к одному из пяти классов паттернов (рис.6).

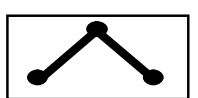

Максимум

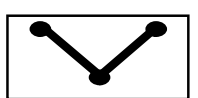

Минимум

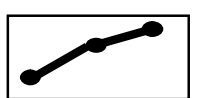

Возрастание

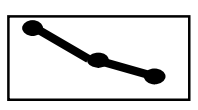

Убывание

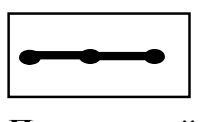

Постоянный

Рис. 6. Классы паттернов предлагаемой перестановочной энтропии при $m=3$.

\section{РЕЗУЛЬТАТЫ ЭКСПЕРИМЕНТОВ НА МОДЕЛЬНЫХ И РЕАЛЬНЫХ ДАННЫХ}

Оценка свойств реализованных вычислительных процедур проводилась на тестовом сигнале в виде модели авторегрессии:

$$
X_{k}=a X_{k-1}+(1-a) \xi_{k-1}, k \in[0 ; N-1]
$$

с начальным значением $X_{0}=0,1$, в которой $a$ - параметр настройки $(0 \leq a \leq 1), N=1000$ - число дискретных отсчетов, $\xi \in N\left(0, \sigma^{2}\right)$ - вектор независимых нормально распределенных случайных величин с нулевым математическим ожиданием и ограниченной дисперсией $\sigma^{2}=10^{2}$ (рис. 7).

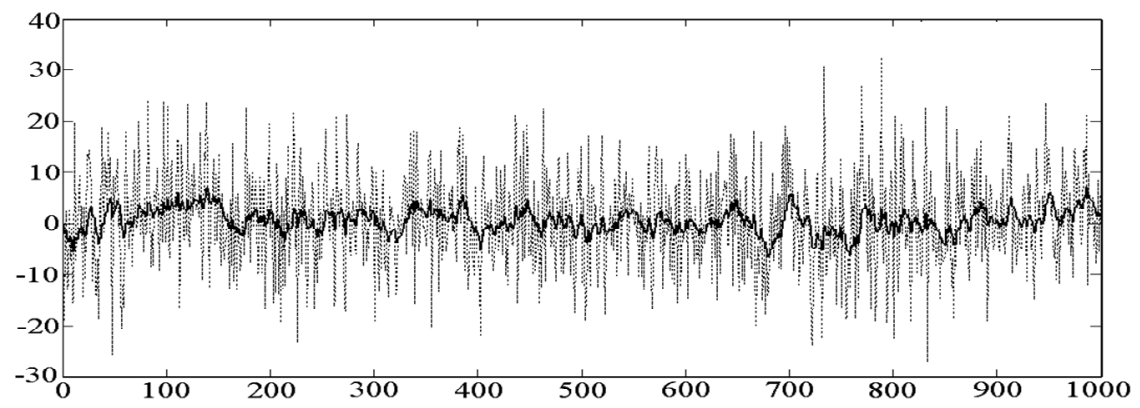

Рис. 7. Примеры модельного сигнала (31) с параметрами $a=0,9$ (черный) и $a=0,1$ (серый)

Усредненные результаты анализа сигналов в серии испытаний с различными параметрами настройки приведены в табл. 1. Значения показателей хаотичности сигнала, которые вычислялись при уменьшении параметра $a$, рассчитывались как прирост ( $\Delta x$, в процентах) к базовому уровню - значению $x$ показателя хаотичности при параметре $a=0,9$. 
Таблица 1.

Усредненные результаты оценки хаотичности модельного сигнала

\begin{tabular}{|c|c|c|c|c|c|c|c|c|c|c|}
\hline \multirow{3}{*}{ Показатели } & \multicolumn{10}{|c|}{ Параметр хаотичности } \\
\hline & \multicolumn{2}{|c|}{$a=0,9$} & \multicolumn{2}{|c|}{$a=0,7$} & \multicolumn{2}{|c|}{$a=0,5$} & \multicolumn{2}{|c|}{$a=0,3$} & \multicolumn{2}{|c|}{$a=0,1$} \\
\hline & $\begin{array}{l}x, \\
\text { ед. }\end{array}$ & $\begin{array}{c}\Delta x, \\
\%\end{array}$ & $\begin{array}{l}x, \\
\text { ед. }\end{array}$ & $\begin{array}{c}\Delta x, \\
\%\end{array}$ & $\begin{array}{l}x, \\
\text { ед. }\end{array}$ & $\begin{array}{l}\Delta x, \\
\%\end{array}$ & $\begin{array}{l}x, \\
\text { ед. }\end{array}$ & $\begin{array}{l}\Delta x, \\
\%\end{array}$ & $\begin{array}{l}x, \\
\text { ед. }\end{array}$ & $\begin{array}{c}\Delta x, \\
\%\end{array}$ \\
\hline $\begin{array}{c}\text { Условная энтропия } \\
E(m \mid m-1)\end{array}$ & 0,73 & 0 & 0,99 & 35,8 & 1,13 & 55,5 & 1,19 & 62,8 & 1,21 & 65,3 \\
\hline $\begin{array}{c}\text { Аппроксимационная } \\
\text { энтропия } A p E n\end{array}$ & 1,06 & 0 & 1,26 & 19,1 & 1,3 & 23 & 1,31 & 24,6 & 1,32 & 25,2 \\
\hline $\begin{array}{c}\text { Энтропия шаблонов } \\
\text { SampEn }\end{array}$ & 1,4 & 0 & 1,86 & 33,8 & 2,05 & 47,2 & 2,16 & 55,2 & 2,18 & 56,3 \\
\hline $\begin{array}{c}\text { Перестановочная } \\
\text { энтропия } P E \\
\end{array}$ & 1,29 & 0 & 1,46 & 14 & 1,49 & 16 & 1,5 & 16,7 & 1,51 & 17,7 \\
\hline Индекс Херста $H_{0}$ & 0,73 & 0 & 0,65 & $-10,4$ & 0,61 & $-16,2$ & 0,58 & $-20,8$ & 0,55 & $-24,7$ \\
\hline
\end{tabular}

Как видно из таблицы, значения всех энтропийных оценок увеличивались по мере увеличения хаотичности сигнала, причем самой чувствительной оказалась условная энтропия $E(m \mid m-1)$. При значении параметра $a=0,9$ показатель Херста принимал значение $H_{0}=0,73$, т.е. принадлежал области $0,5<H_{0} \leq 1$ устойчивых (персистентных) сигналов с признаками самоподобия. Поэтому по мере уменьшения параметра $a$ (увеличения степени хаотичности) индекс Херста, в отличие от энтропийных оценок, уменьшался, что согласуется с результатами других работ, в частности, с работой [23].

Проводились также эксперименты по оценке степени хаотичности искусственных ЭКГ (рис. 8), которые генерировались на основе моделей, приведенных в монографии [24].

Первый сигнал (рис. 8, а) представлял собой последовательность эталонных циклов, у которых хаотически изменялась амплитуда зубца Т в пределах $30 \%$ базового значения $A_{T}=0,2$ мB. Второй сигнал (рис. 8, б) моделировал альтернацию амплитуды зубца $\mathrm{T}$ с уровнем альтернации 60 мкВ.

Таким образом, в первой ЭКГ амплитуда изменялась случайным образом, во втором - строго чередуясь, хотя визуально сигналы практически неразличимы (рис. 8).

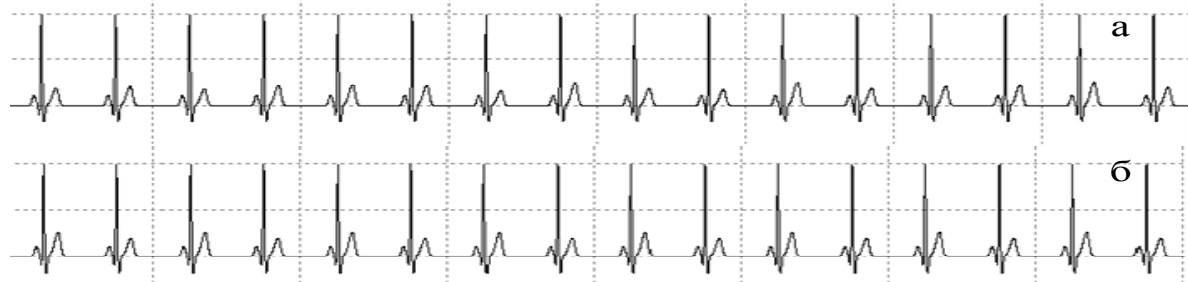

Рис. 8 Искусственная ЭКГ: а) со случайным искажением амплитуды зубца Т; б) с альтернацией (чередованием) амплитуд зубца Т разного уровня 
Заметим, что среднеквадратические отклонения амплитуд зубцов Т на обоих сигналах были схожими: $C K O_{\text {случ }}=0,036$ и $C K O_{\text {альm }}=0,03$. Тем не менее, при оценке энтропий, эти отличия были более выраженными (табл. 2).

Таблица 2.

Результаты обработки искусственных ЭКГ

\begin{tabular}{c|c|c}
\hline \hline \multirow{2}{*}{ Показатели } & \multicolumn{2}{|c}{ Модельный сигнал с искажением зубца т } \\
\cline { 2 - 3 } & Случайное & Альтернация \\
\hline \hline $\begin{array}{c}\text { Условная энтропия } \\
E(m \mid m-1)\end{array}$ & 1,056 & 0 \\
\hline $\begin{array}{c}\text { Аппроксимационная } \\
\text { энтропия } A p E n\end{array}$ & 0,512 & 0,030 \\
\hline $\begin{array}{c}\text { Энтропия шаблонов } \\
\text { SaтрEn }\end{array}$ & 1,5041 & 0 \\
\hline $\begin{array}{c}\text { Перестановочная } \\
\text { энтропия } P E\end{array}$ & 1,485 & 0,693 \\
\hline Индекс Херста $H_{0}$ & 0,525 & 0 \\
\hline \hline
\end{tabular}

Далее рассмотрим результаты оценки хаотичности реальных сигналов (рис. 9) - двух ритмограмм (100 последовательных $R-R$ интервалов), отражающих ритм сердца спортсмена (рис. 9, а) и не спортсмена (рис. 9, б) одного возраста (20 лет) сразу после физической нагрузки (20 глубоких приседаний за 30 сек).

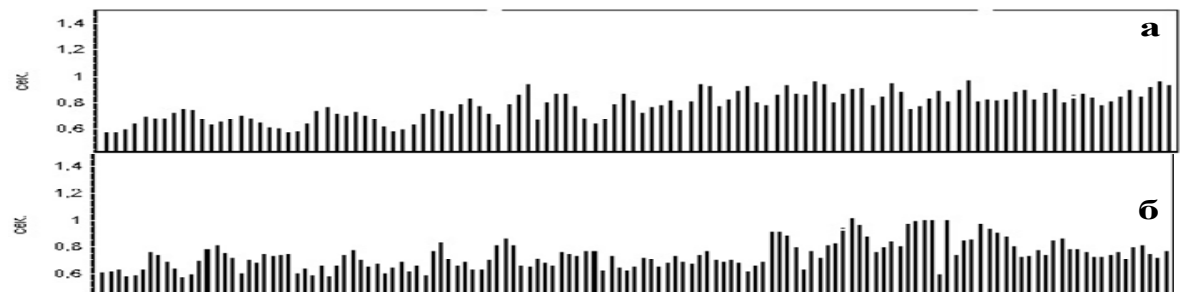

Рис. 9. Ритмограммы: а) спортсмена, б) человека, не занимающегося спортом

Хотя обе ритмограммы имеют практически одинаковые среднеквадратические отклонения ( $S D N N=105)$, при оценке хаотичности этих сигналов наблюдаются расхождения ряда показателей (табл. 3).

Таблица 3.

Показатели хаотичности ритма сердиа после физической нагрузки

\begin{tabular}{c|c|c|c}
\hline \hline Показатели & Спортсмен & Не спортсмен & Отличия, \% \\
\hline \hline $\begin{array}{c}\text { Условная энтропия } \\
E(m \mid m-1)\end{array}$ & 0,559 & 0,670 & 19,9 \\
\hline $\begin{array}{c}\text { Аппроксимационная } \\
\text { энтропия } A p E n\end{array}$ & 0,535 & 0,617 & 15,3 \\
\hline $\begin{array}{c}\text { Энтропия шаблонов } \\
\text { SampEn }\end{array}$ & 1,017 & 1,034 & 2,8 \\
\hline $\begin{array}{c}\text { Перестановочная } \\
\text { энтропия } P E\end{array}$ & 1,350 & 1,351 & 0,07 \\
\hline Индекс Херста $H_{0}$ & 0,791 & 0,761 & $-3,8$ \\
\hline \hline
\end{tabular}

(С) Л.С. Файнзильберг, К.Б. Ориховская, И.В. Ваховский, 2016 
На рис. 10 представлены ритмограмма пациентки А. 67 лет (рис. 10, а), у которой наблюдалась умеренная аритмия $(S D N N=63)$ и ритмограмма практически здорового человека В. (рис. 10, б) в возрасте 31 года с практически таким же значением показателя вариабельности ритма сердца $(S D N N=50)$.

Характерная особенность ритмограммы пациентки А. (рис. 10, а) альтернация трех значений продолжительности $R-R$ интервалов, которые последовательно чередуются друг за другом. На ритмограмме пациента $\mathbf{B}$. (рис. 10,6 ) такие особенности отсутствуют. Заметим, что указанные отличия практически не заметны на исходных ЭКГ (рис. 11), по которым строились ритмограммы.

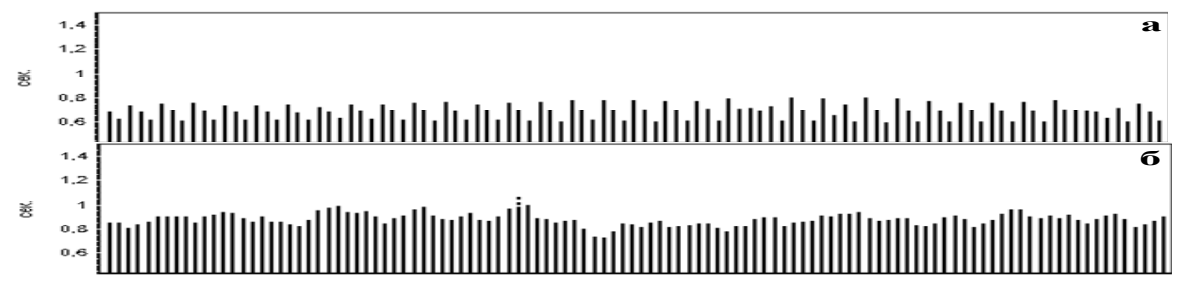

Рис. 10. Ритмограммы: а) пациентки А., б) практически здорового пациента В.

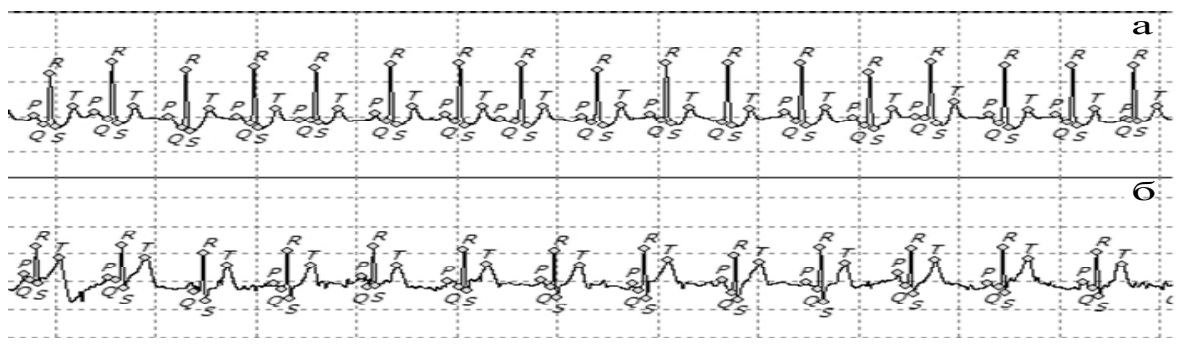

Рис. 11. Исходные ЭКГ: а) - пациентки А., б) здорового пациента В.

В то же время и в данном случае наблюдались существенные отличия показателей хаотичности этих ритмограмм (табл. 4).

Таблица 4.

Показатели хаотичности ритма сердй пациентов

\begin{tabular}{|c|c|c|c|}
\hline Показатели & Пациент B. & Пациентка А. & Отличия, \% \\
\hline $\begin{array}{c}\text { Условная энтропия } \\
E(m \mid m-1)\end{array}$ & 0,713 & 0,530 & $-25,7$ \\
\hline $\begin{array}{l}\text { Аппроксимационная энтропия } \\
\qquad A p E n\end{array}$ & 0,533 & 0,301 & $-43,5$ \\
\hline $\begin{array}{l}\text { Энтропия шаблонов } \\
\text { SampEn }\end{array}$ & 1,142 & 0,348 & $-69,5$ \\
\hline $\begin{array}{c}\text { Перестановочная } \\
\text { энтропия } P E \\
\end{array}$ & 1,323 & 1,261 & $-4,7$ \\
\hline Индекс Херста $H_{0}$ & 0,688 & 0,538 & $-21,8$ \\
\hline
\end{tabular}

Как видно, показатели энтропии у пациентки А. существенно ниже, чем у пациента В. Это свидетельствует о том, что, в отличие от ритмограммы 
здорового пациента, ритмограмма с альтернацией имеет четко выраженную регулярную составляющую.

Индекс Херста у здорового пациента В. был на $22 \%$ выше, чем у пациентки А. Этот факт также согласуется с данными работы [25], в которой отмечается, что более низкий уровень индекса Херста несет информацию об ухудшении физиологических или патофизиологических процессов в работе сердечно-сосудистой системы.

В завершение приведем интересные результаты по сравнительной оценке степени хаотичности показателей ЭКГ, несущих информацию об изменении формы фрагментов ЭКГ, в двух группах испытуемых (табл. 5).

\section{Таблица 5.}

Различия средних значений показателей хаотичности формы фрагментов ЭКГ в двуx грynnax, $(x \pm S x), n=70$

\begin{tabular}{c|c|c|c}
\hline \hline \multicolumn{2}{c|}{ Показатель формы фрагмента ЭкГ } & \multicolumn{2}{c}{ Степень хаотичности показателя } \\
\hline Наименование & Обозначение & $\begin{array}{c}\text { Группа 1 } \\
\text { (спортсмены) }\end{array}$ & $\begin{array}{c}\text { Группа 2 } \\
\text { (не спортсмены) }\end{array}$ \\
\hline \hline Правосторонний наклон зубца $P$ & $b_{P}(2)$ & $1,52 \pm 0,24 * *$ & $1,71 \pm 0,28$ \\
\hline Момент появления зубца $P$ & $\mu_{P}$ & $1,26 \pm 0,3 * *$ & $1,48 \pm 0,37$ \\
\hline Амплитуда зубца $Q$ & $A_{Q}$ & $2,12 \pm 0,39^{*}$ & $1,94 \pm 0,41$ \\
\hline Левосторонний наклон зубца $Q$ & $b_{Q}(1)$ & $1,5 \pm 0,28^{*}$ & $1,68 \pm 0,29$ \\
\hline Амплитуда зубца $R$ & $A_{R}$ & $2,11 \pm 0,45^{*}$ & $1,85 \pm 0,48$ \\
\hline Момент появления зубца $R$ & $\mu_{R}$ & $1,11 \pm 0,34 * *$ & $0,87 \pm 0,33$ \\
\hline Амплитуда зубца $S$ & $A_{S}$ & $1,82 \pm 0,26^{*}$ & $1,61 \pm 0,47$ \\
\hline Момент появления зубца $S$ & $\mu_{S}$ & $1,15 \pm 0,35^{*}$ & $0,91 \pm 0,41$ \\
\hline Момент появления сегмента $S T$ & $\mu_{S T}$ & $1,51 \pm 0,3^{* * *}$ & $1,14 \pm 0,4$ \\
\hline Амплитуда зубца $T$ & $A_{T}$ & $1,96 \pm 0,45^{*}$ & $1,61 \pm 0,64$ \\
\hline Левосторонний наклон зубца $T$ & $b_{T}(1)$ & $1,93 \pm 0,28^{* *}$ & $1,71 \pm 0,32$ \\
\hline Правосторонний наклон зубца $T$ & $b_{T}(2)$ & $2,08 \pm 0,4^{* *}$ & $1,81 \pm 0,39$ \\
\hline Симметрия зубца $T$ & $\mu_{T}$ & $1,22 \pm 0,35^{* * *}$ & $0,79 \pm 0,35$ \\
\hline \hline
\end{tabular}

Примечание: достоверность различий по критерию Стьюдента: * $p<0,05 ; * * p<0,01 ; * * * p<0,001$

Группы состояли из молодых волонтеров обоего пола в возрасте 18-24 лет. Группа 1 включала 28 спортсменов высшей квалификации, которые занимаются боксом, разными видами борьбы и триатлоном. В группу 2 вошли 42 человека, не занимающихся спортом. Анализ хаотичности сигнала (100 последовательных кардиоциклов ЭКГ 
каждого испытуемого) проводился сразу после физической нагрузки (20 глубоких приседаний за 30 сек.) с помощью энтропии шаблонов SampEn по формуле (15). Достоверность отличий в группах оценивалась по критерию Стьюдента.

Как видно из таблицы, у тренированных и нетренированных лиц наблюдаются достоверные отличия хаотичности ряда показателей, характеризующих форму фрагментов ЭКГ. При этом у тренированных лиц хаотичность одних показателей под нагрузкой была выше, чем у нетренированных, а других показателей - ниже.

Обнаруженные факты, вероятно, отражают различия в степени «обученности» отдельных участков миокарда спортсменов и не спортсменов к выполнению нагрузок. Разумеется, обнаруженные явления, которые могут открыть новые возможности в оценке адекватности реакции организма на нагрузку, требуют дальнейших углубленных исследований с участием физиологов и спортивных врачей.

\section{Выводы}

Проведенный анализ существующих подходов к оценке хаотичности временных рядов показывает, что современная наука располагает широким арсеналом методов и вычислительных алгоритмов, позволяющих с различных сторон оценить степень хаотичности временных рядов, порождаемых медико-биологическими системами.

Для практического применения этих алгоритмов разработана инструментальная система, обеспечивающая проведение необходимых исследований при разработке новых медицинских систем, выбор подходящего вычислительного алгоритма, удобство настройки алгоритма под конкретные данные и принятие решения о необходимости модификации алгоритма.

Представленные результаты экспериментальных исследований на модельных и реальных данных подтвердили, что реализованные в инструментальной системе вычислительные алгоритмы позволяют адекватно оценивать степень хаотичности биологических сигналов - ЭКГ и ритмограмм.

Установлено, что при оценке хаотичности показателей формы элементов ЭКГ удается выявить диагностически важные, тонкие изменения этих сигналов здоровых и больных пациентов, а также достоверные различия показателей хаотичности элементов формы ЭКГ у людей с различной степенью тренированности организма.

1. Тузов В.В. Методы синергетики // Библиосфера. — 2009. — № 4. - С. 8-14.

2. Modified permutation-entropy analysis of heartbeat dynamics / C. Bian, C. Qin, Q.D. Ma \& others // Physical Review E. - 2012. - №. 85.

3. Classifying cardiac biosignals using ordinal pattern statistics and symbolic dynamics / U. Parlitz, S. Luther Berg, S. Schirdewan \& others // Computers in Biology and Medicine. 2012. — №. 42. - P. 319-327. 
4. Permutation entropy improves fetal behavioural state classification based on heart rate analysis from biomagnetic recordings in near term fetuses / B. Frank, B. Pompe, U. Schneider \& others // Medical \& Biological Engineering \& Computing. - 2006. №. 44. - P. 179-187.

5. Изучение зависимостей между показателями вариационной пульсометрии, энтропии ритма сердца, временного и спектрального анализов вариабельности ритма сердца в норме и при ишемической болезни сердца / Н.Ю. Дурнова, Я.П. Довгалевский, А.Н. Бурлака и др. // Саратовский научно-медицинский журнал. — 2011. — том 7. № 3. - С. 608-611.

6. Вентцель Е.С. Теория вероятностей: учебник для вузов. — М.: Высшая школа. - 1999. $-576 \mathrm{c}$.

7. Кузнецов А.А. Методы анализа и обработки электрокардиографических сигналов: новые подходы к выделению информации: монография. - В.: ВлГУ. - 2008. - 140 с.

8. Сотников П.И. Выделение характерных признаков сигнала электроэнцефалограммы с помощью анализа энтропии // Наука и Образование. — 2014. — № 11. - С. 555-570.

9. Сравнительный анализ применения различных оценок энтропии ЭЭГ-сигнала для распознавания стадий наркоза / А.П. Немирко, Л.А. Манило, А.Н. Калиниченко и др. // Биотехносфера. - 2010. - № 3. - С. 3-10.

10. Takens F. Detecting strange attractors in turbulence // Dynamical systems and turbulence: lecture notes in mathematics. — 1981. - Vol. 898. - P. 366-381.

11. Pincus S.M. Approximate entropy as a measure of system complexity // Proceedings of the National Academy of Sciences. - 1991. — Vol. 88. - P. 2297-2301.

12. Данильчук А.Б. Использование энтропийных показателей для моделирования динамики сложных социально-экономических систем // Economics. — 2014. — № 3. C. $19-24$.

13. Юшковская О.Г. Новый подход к оценке эффективности санаторно-курортной реабилитации больных ишемической болезнью сердца // Физкультура в профилактике, лечении и реабилитации. - 2004. - № 1. - С. 22-26.

14. Costa M., Ary L., Goldberger A.L. Multiscale entropy analysis of biological signals // Physical Review E. — 2005. - №. 71.

15. Pincus S.M, Goldberger A.L. Physiological time-series analysis: what does regularity quantify? // The American journal of physiology. — 1994. — Vol. 266. — P. 1643-1656.

16. Joshua S., Richman J., Moorman R. Physiological time-series analysis using approximate entropy and sample entropy // The American journal of physiology. — 2000. - Vol. 278. №. 6. - P. 2039-2049

17. Bandt C., Pompe B. Permutation entropy - a natural complexity measure for time series // Chinese Physics B. - 2001. - Vol. 18. - №. 7. - P. 2690-2696.

18. Антипов И.Е., Захаров А.В., Повереннова О.И. Возможности различных методов автоматического распознавания стадий сна // Саратовский научно-медицинский журнал. — 2012. — том 8. - № 2. - С. 374-379.

19. Мун Ф. Хаотические колебания: Вводный курс для научных работников и инженеров: Пер. с англ. - М.: Мир. - 1990. - 312 с.

20. Майоров О.Ю., Фенченко В.Н. Повышение надежности исследований детерминированного хаоса в биоэлектрической активности (ЭЭГ, ЭКГ и вариабельности сердечного ритма) методами нелинейного анализа // Клиническая информатика и телемедицина. — 2009. — том 6. — № 6. - С. 10-17.

21. Киселева О.Г., Настенко Е.А., Герасимчук М.В. Метод оценки дизадаптационных состояний организма человека // Восточно-Европейский журнал передовых технологий. - 2011. - том 3. - № 2. - С. 57-64.

22. Зиненко А.В. R/S анализ на фондовом рынке // Бизнес-информатика. — 2012. №3. - C. 24-30.

23. Апанасенко Г.Л., Чистякова Ю. С. Здоровье спортсмена: критерии оценки и прогнозирования // Теория и практика физической культуры. - 2006. - № 1. C.19-22. 
24. Файнзильберг Л.С. Компьютерная диагностика по фазовому портрету электрокардиограммы. - Киев: Освита Украины. — 2013. — 191 с.

25. Способ диагностики функционального состояния человека и животного: пат. 2254051 Россия: МПК А61В005/02 / Загускин С.Л., Борисов В.А. Заявлено 02.10.03; Опубл. 20.06.2005 // Бюл. — 2006. — № 13.

UDC 616.12

\title{
ASSESSMENT OF CHAOTIC FRAGMENTS' SHAPE OF THE SINGLE-CHANNEL ELECTROCARDIOGRAM
}

\author{
L.S. Fainzilberg ${ }^{1}$, K.B. Orikhovska ${ }^{1}$, I.V. Vakhovskyi ${ }^{2}$ \\ ${ }^{1}$ International Research and Training Center for Information Technologies and \\ Systems of National Academy of Sciences of Ukraine and Ministry of Education and \\ Science of Ukraine (Kiev) \\ ${ }^{2}$ National Technical University of Ukraine "Kiev Polytechnical Institute” (Kiev)
}

Introduction. Building an effective information technology (IT), which provides chaotic assessment of the electrocardiogram (ECG) fragments' shape, has both cognitive and practical importance. Therefore, the problem of developing methods and computer tools that provide assessment not only by the rhythm of the heart, but also on other parameters of ECG that have diagnostic value is relevant.

The purpose of the article is to propose instrumental system for the study of single-channel ECG elements shape chaoticity, based on the various entropy assessments and make a comparative analysis of these estimates in the model and the real data.

Methods. The proposed instrumental system based on the national portable electrocardiograph FAZEGRAF ${ }^{\circledR}$ with the original finger electrodes sensor, which can record the ECG from the first standard lead. In addition to determining the chaoticity of parameters, that characterize the shape of the main elements of the ECG, also estimating the diagnostic features chaoticity. Introduced a number of improvements in the considered methods which determine the signal chaoticity degree. In particular, an original evaluation algorithm for permutation entropy evaluating that can automatically identify 5 classes of patterns is proposed.

Results. Processing of model and real data showed that the computing algorithms implemented in IT allow to adequately assessing the degree of signals chaoticity. Based on the parameters chaotic assessment, that carry information about the ECG elements shape, diagnostically important subtle signal differences in healthy and sick patients, as well as significant differences in parameters of the ECG elements shape chaoticity in people with varying degrees of organism fitness were found.

Conclusions. Instrumental system provides the convenience of experimental studies with searching for new biomarkers of cardiac abnormalities and evaluation of organism adaptation capabilities.

Keywords: heart rate, the entropy of the process, synergy, shape of ECG fragments. 
1. Tuzov V.V. Methods of synergetics // Bibliosphere. - 2009. — №. 4. - P. 8-14. (in Russian).

2. Modified permutation-entropy analysis of heartbeat dynamics / C. Bian, C. Qin, Q.D. Ma \& others // Physical Review E. — 2012. - №. 85.

3. Classifying cardiac biosignals using ordinal pattern statistics and symbolic dynamics / U. Parlitz, S. Luther Berg, S. Schirdewan \& others // Computers in Biology and Medicine. 2012. - №. 42. - P. 319-327.

4. Permutation entropy improves fetal behavioural state classification based on heart rate analysis from biomagnetic recordings in near term fetuses / B. Frank, B. Pompe, U. Schneider \& others // Medical \& Biological Engineering \& Computing. — 2006. №. 44. - P. $179-187$.

5. The study of relationships between parameters of variation pulsometry, entropy of heart rate, time and spectral analysis of heart rate variability in normal and ischemic heart disease / N.Y. Durnova, Y.P. Dovgalevskij, A.N. Burlaka \& others // Saratov Medical Scientific Research Journal - 2011. — Vol. 7. — №. 3. - P. 608-611. (in Russian).

6. Wentsel E.S. Theory of probability. — Moskow: Science. - 1969. - 575 p. (in Russian).

7. Kuznecov A.A. Methods of analysis and processing of ECG signals: new approaches to information extraction: monograph. - Vladimir: Vladimir State University Publishing. 2008. - 140 p. (in Russian).

8. Sotnikov P.I. Isolation of the characteristic features of a EEG signal by the entropy analyzing // Science and Education. - 2014. — №. 11. - P. 555-570. (in Russian).

9. Comparative analysis of the different estimates usage of the EEG signal entropy for recognizing the anesthesia stages / A.P Nemirko, L.A. Manilo, A.N. Kalinichenko \& others // Biotechnosphere. - 2010. - №. 3. - P. 3-10. (in Russian).

10. Takens F. Detecting strange attractors in turbulence // Dynamical systems and turbulence: lecture notes in mathematics. — 1981. — Vol. 898. - P. 366-381.

11. Pincus S.M. Approximate entropy as a measure of system complexity // Proceedings of the National Academy of Sciences. — 1991. - Vol. 88. — P. 2297-2301.

12. Danilchuk A.B. Using entropy parameters for simulating the dynamics of complex social and economic systems // Economics. — 2014. — №. 3. - P. 19-24. (in Russian).

13. Jushkovskaja O.G. A new approach to assessing the effectiveness of sanatorium rehabilitation of patients with coronary heart disease // Physical education in the prevention, treatment and rehabilitation. - 2004. - №. 1. - P. 22-26. (in Russian).

14. Costa M., Ary L., Goldberger A.L. Multiscale entropy analysis of biological signals // Physical Review E. - 2005. - №. 71.

15. Pincus S.M., Goldberger A.L. Physiological time-series analysis: what does regularity quantify? // The American journal of physiology. — 1994. - Vol. 266. - P. 1643-1656.

16. Joshua S. Richman J., Moorman R. Physiological time-series analysis using approximate entropy and sample entropy // The American journal of physiology. — 2000. - Vol. 278. №. 6. - P. 2039-2049.

17. Bandt C., Pompe B. Permutation entropy - a natural complexity measure for time series // Chinese Physics B. — 2001. — Vol. 18. — №. 7. - P. 2690-2696.

18. Antipov I.E., Zakharov A.V., Poverennova O.I. The possibilities of different methods of automatic recognition of sleep stages // Saratov Medical Scientific Research Journal. 2012. - Vol. 8. - №. 2. - P. 374-379. (in Russian).

19. Mun F. Chaotic oscillations: Introductory course for scientists and engineers: Trans. from English. - Moskow: Mir. - 1990. — 312 p. (in Russian).

20. Mayorov O.Y., Feshchenko V.N. Improving the reliability of studies of deterministic chaos in the bioelectric activity (EEG, ECG and heart rate variability) methods of nonlinear analysis // Clinical Informatics and Telemedicine. — 2009. - Vol. 6. — № 6. - P. 10-17. (in Russian).

21. Kiseleva O.G., Nastenko Ie.A., Gerasimchuk M.V. Method of estimation disadaptation states of the human body // East European Journal of advanced technologies. - 2011. Vol. 3. - №. 2. - P. 57-64. (in Russian). 
22. Zinenko A.V. R/S analysis of the stock market // Business Informatics. - 2012. - № 3. P. 24-30. (in Russian).

23. Apanasenko G.L., Chistyakova Yu. S. Athlete's Health Criteria evaluation and prediction // Theory and Practice of Physical Culture. - 2006. - № 1. - P.19-22. (in Russian).

24. Fainzilberg L.S. Computer diagnostics by phase portrait of electrocardiogram. - Kiev: Osvita Ukrainy. - 2013. - 191 p. (in Russian).

25. Zaguskin S.L., Borisov V.A. A method for diagnosing the functional state of human and animal. RU Patent 2254051. — 2006. — bul. 13. (in Russian).

Получено 08.12.2015 